\title{
La evaluación de la educación virtual: las e-actividades
}

\section{(The Evaluation of Virtual Education: e-Activities)}

\author{
Julio Cabero-Almenara \\ Antonio Palacios-Rodríguez \\ Universidad de Sevilla (España)
}

DOI: https://doi.org/10.5944/ried.24.2.28994

\section{Cómo referenciar este artículo:}

Cabero-Almenara, J., y Palacios-Rodríguez, A. (2021). La evaluación de la educación virtual: las e-actividades. RIED. Revista Iberoamericana de Educación a Distancia, 24(2), pp. 169-188. https://doi.org/10.5944/ried.24.2.28994

\section{Resumen}

Una de las fases del proceso de instrucción que menos cambios ha sufrido a lo largo de la historia ha sido la de la evaluación. En un contexto virtual, una de las preocupaciones que muestran los docentes respecto a la evaluación se relaciona con las problemáticas de su implementación y la vertebración de diferentes e-actividades. Por e-actividades se entienden todas las tareas desarrolladas por el estudiante de forma individual o colectiva en un entorno digital, y que están destinadas a obtener un aprendizaje específico. Son, por tanto, el vínculo que une la enseñanza y el aprendizaje en la denominada Sociedad del Conocimiento. En esta línea, este artículo trata de realizar una panorámica por el fenómeno de las e-actividades dentro de los entornos virtuales de aprendizaje. Para ello, realiza una síntesis de los principales estudios sobre el tema en cuanto a definición, tipología, selección y análisis. Los resultados ofrecen un recorrido que avala la importancia de estos recursos como objetos de aprendizaje auténtico. Por este motivo, es necesario que los docentes desarrollen competencias en cuanto a su selección, creación, modificación y evaluación. En esta línea, se esbozan diferentes criterios a tener en cuenta y que se pueden movilizar para el desarrollo de dichas competencias clave.

Palabras clave: educación virtual; evaluación; e-actividades; formación del profesorado; competencia digital docente.

\begin{abstract}
One of the phases of the instructional process that has undergone minor changes throughout history has been that of evaluation. In a virtual context, one of the concerns that teachers show regarding evaluation is related to the problems of its implementation and the
\end{abstract}


design, organisation and eventually implementation of different e-activities. By e-activities we understand all the tasks developed by a student individually or collectively in a digital environment, and which are intended to obtain specific learning. They are, therefore, the link that unites teaching and learning in the so-called Knowledge Society. Along these lines, this article offers an overview of the phenomenon of e-activities within virtual learning environments. Accordingly, it goes through the main studies on e-activities in terms of definition, typology, selection and analysis. Results obtained show the relevance of such resources as authentic learning objects and highlight the importance of promoting specific teacher training competences on the selection, creation, modification and evaluation of e-activities. In this line, the present study proposes different criteria to be considered for the effective inclusion of such competences in teachers' training programmes on digital teaching competence.

Keywords: virtual education; evaluation; e-activities; teacher training; digital teaching competence.

En los últimos tiempos, la educación ha tenido una diversidad de cambios en lo referido a la aplicación de nuevas metodologías y estrategias de formación: aplicación de metodologías activas, gamificación, flipped classroom... Dichos cambios también han ocurrido en la incorporación de nuevas tecnologías; desde las ya tradicionales como Internet a las emergentes como la realidad aumentada o las analíticas de aprendizaje. Por ello, es necesaria una nueva fundamentación con teorías del aprendizaje construidas, como el conectivismo o el aprendizaje rizomático, para fundamentar el aprendizaje en red.

Sin embargo, en contraposición a todo esto, no ha ocurrido lo mismo con una de las fases más significativas del proceso de instrucción, la evaluación. Los procesos evaluativos siguen apoyándose en concepciones tradicionales y en los mismos instrumentos, como ha mostrado el momento del confinamiento y la inseguridad que ha mostrado gran parte del profesorado para llevar a cabo nuevas modalidades evaluativas soportadas en la red (Moorhouse, 2020).

Como señalan Blázquez et al. (2017, p. 25), "si dentro de la cultura de la escuela existe una dimensión que revela mejor la existencia de creencias, mitos, ritos y costumbres que se resisten al cambio, esa es la evaluación”. Estos aspectos vienen motivados, como señalan los autores anteriormente mencionados, por una enseñanza que es entendida como "transmisión de información, que suele conducir a un tipo de aprendizaje acrítico y mecánico, así como a una evaluación que busca verificar la capacidad del alumno para responder de memoria a las preguntas o enunciados formulados por el docente" (Blázquez et al., 2017, p. 25).

Lo comentado lleva a señalar que una de las preocupaciones que muestran los docentes cuando se trabaja en una modalidad virtual de enseñanza respecto a la evaluación se relaciona con las problemáticas de la suplantación de la persona y la 
copia y plagio de la información en lugar de otras relacionadas con el desarrollo de competencias clave (Sangrá, 2020).

Bajo esta perspectiva, la evaluación pierde su verdadero sentido, mejorar el proceso de instrucción sirviendo, además de para conocer el dominio sobre los contenidos y la adquisición de competencias por el estudiante; y para analizar la eficacia de la metodología aplicada, los recursos utilizados y la estructura organizativa utilizada (Marcano et al., 2020). En otros términos, la finalidad de la evaluación, independientemente de su tipología (diagnóstica, formativa o sumativa), es mejorar el Sistema Educativo. Como señalan Lezcano y Vilanova, "en función de cómo la evaluación sea considerada al diseñar el proceso, puede ser percibida como un juicio o como una ocasión para aprender" (2017, p. 2). El problema es que, habitualmente, "la evaluación suele representar el final de un proceso de aprendizaje, por lo que no suele tener repercusiones directas sobre el aprendizaje" (Ruiz-Morales, 2020, p. 279). De ahí que buscar nuevos modelos de evaluación como el apoyado en tareas y actividades pueden ser de gran utilidad para potenciar/mejorar el proceso de enseñanza-aprendizaje.

Todo ello lleva a inferir que la evaluación no se refiere únicamente a la administración de instrumentos para indagar sobre lo aprendido o recordado por parte del estudiante, sino más bien a un planificado proceso de recogida de información que permita analizar el proceso formativo diseñado.

Para Ruiz-Morales (2008), independientemente de la noción de evaluación que se adopte, existen una serie de puntos comunes:

1. La evaluación como algo necesario en el proceso de enseñanza y aprendizaje.

2. Proceso de valoración sistemática.

3. Recogida de datos.

4. Análisis de los datos.

5. Emisión de juicios de valor.

6. Toma de decisiones.

Frente a modelos de evaluación preocupados únicamente en la recogida de la información en los que el alumnado es capaz de recordar de los contenidos que se le han presentado, y que sugieren una metodología transmisiva por parte del docente; en la actualidad se defienden nuevas modalidades de formación en los entornos virtuales, donde la actividad realizada es clave para la adquisición del conocimiento. Por supuesto, este fenómeno conlleva pasar de un modelo de formación centrado en el profesor a otro centrado en el estudiante. De la misma forma, lleva a asumir los siguientes principios:

1. Pasar del alumno como receptor a desarrollador.

2. De entornos formativos basados en la recepción a la participación einteractividad. 
3. De centrarnos en productos a procesos centrados en el desempeño y la competencia.

4. Desplazarnos de una evaluación sumativa a una evaluación auténtica.

5. Intercambio en clase al intercambio en la comunidad y el apoyo en la interactividad.

Para Cabrera y Fernández-Ferrer (2020), tres son las agrupaciones de estrategias entre las que podemos elegir para la realización de la evaluación on-line:

1. Estrategias y herramientas que permiten acreditar la comprensión de los contenidos, entre las que se encuentran los cuestionarios en-línea.

2. Las que promueven el desarrollo competencial a través de la realización de actividades.

3. La gamificación.

De forma complementaria, como señalan Medina-Díaz y Verdejo-Carrión (2020, p. 271), la utilización de las

metodologías activas o auténticas, como estrategias de enseñanza, sirven de vehículo para facilitar su desarrollo y logro, mediante situaciones o problemas similares a los que se confrontan en el campo profesional y la sociedad. Su aplicación, requiere replantear, planificar y orientar la enseñanza de modos distintos, ubicando al o la estudiante como centro, y alineando las técnicas y los instrumentos para la evaluación.

Estas metodologías activas sitúan en el centro del proceso formativo al estudiante para generar un aprendizaje situado (Silva, 2017).

Asociada a dichas metodologías, se encuentra el concepto de evaluación auténtica (Cubero-Ibáñez y Ponce-González, 2020; Barrientos-Hernán et al., 2020; Brown, 2015). La evaluación auténtica, como señala Brown (2015, p. 3),

tiene lugar cuando examinamos directamente el desempeño de los estudiantes en tareas intelectuales relevantes, cuando los estudiantes están obligados a ser intérpretes eficaces de los conocimientos adquiridos y podemos hacer inferencias válidas sobre el desempeño del estudiante a partir de las tareas que se utilizan para la evaluación.

$\mathrm{Al}$ respecto, se encuentra la utilización de tareas, actividades y estrategias que llevan a "pensar creativamente y a veces lateralmente" (Brown, 2015, p. 4). Además, "alientan a los estudiantes a enfrentarse a prácticas desafiantes, proporcionando oportunidades, a través de las formas en que estos participan en los procesos de evaluación, para que aprendan y reflexionen sobre su aprendizaje favoreciendo así 
su desarrollo personal y profesional” (Cubero-Ibáñez y Ponce-González, 2020, p. 42). No se debe olvidar que estas tareas están estrechamente relacionadas con la evaluación continua y con la transferencia del aprendizaje (Díaz Rosas et al., 2019).

A continuación, se analiza el concepto de actividades o tareas en un contexto de formación virtual, las denominadas e-actividades.

\section{LAS E-ACTIVIDADES EN LA FORMACIÓN VIRTUAL}

Diversas son las variables y competencias que el docente debe manejar en la formación virtual (Cabero-Almenara y Palacios-Rodríguez, 2020; Padilla-Hernández et al., 2019). Una de ellas son las e-actividades que los estudiantes deben realizar y las estrategias de evaluación relacionadas con las mismas. Éstas, como se esquematiza en la figura 1, repercuten en crear un entorno formativo de calidad.

\section{Figura 1}

Variables críticas de la formación virtual

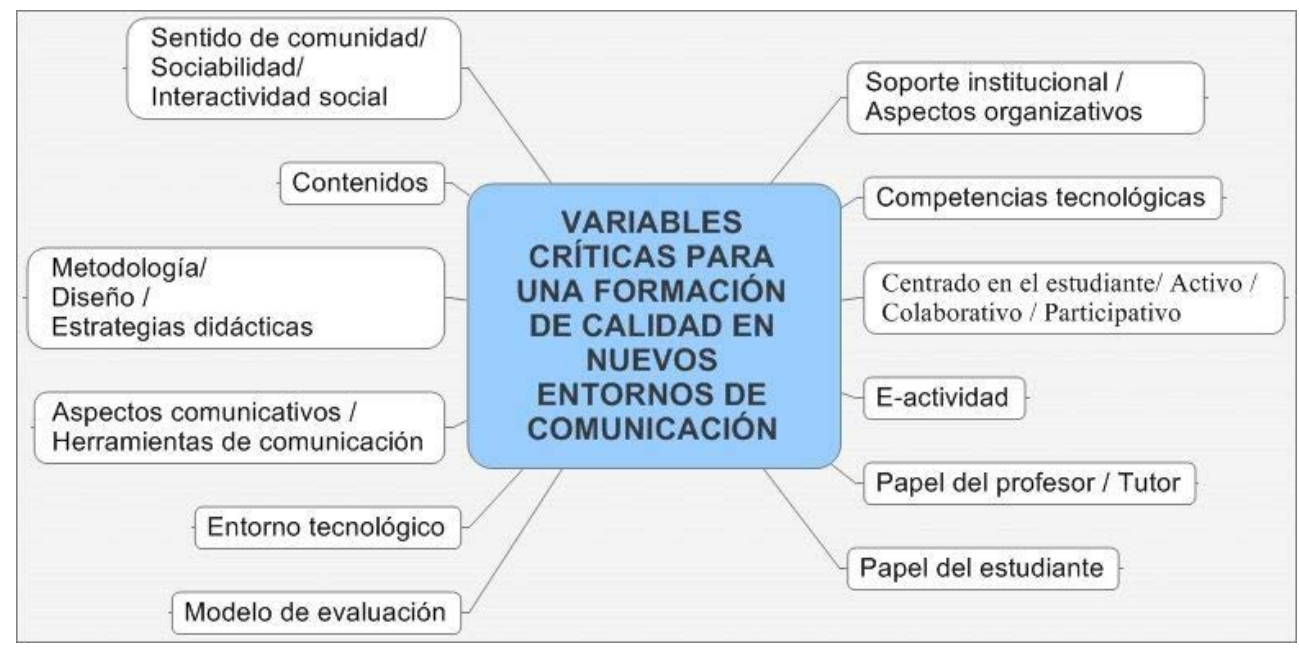

Dichas variables inducen el aprendizaje en los estudiantes. Conjuntamente, llevan al docente a diseñar acciones formativas más centradas en el aprendizaje que en la enseñanza, y hacen que el alumnado reflexione y pueda poner en acción la información y los contenidos que se le han presentado. Por otra parte, cuando se han realizado diferentes investigaciones sobre los aspectos más significativos que perciben los profesores y los estudiantes, se perciben como uno de los elementos que garantiza la calidad de la formación virtual (Lai et al., 2017). 
Dentro del concepto de e-actividades, se comienza con la reflexión que indican Fuentes-Guerra y García (2003, p. 147) cuando señalan que el "concepto de actividad en el proceso de enseñanza aprendizaje es muy genérico y no tiene que ir obligatoriamente asociado a movimiento o acto manipulativo del alumnado, sino que se refiere a todas aquellas acciones (de observación, escucha, trabajo en equipo...) que nos lleven a facilitar el proceso de enseñanza-aprendizaje”.

Con base en la anterior reflexión, por e-actividades se entienden todas las tareas desarrolladas por el estudiante de forma individual o colectiva en un entorno digital, y que están destinadas a obtener un aprendizaje específico. Son, por tanto, el vínculo que une la enseñanza y el aprendizaje en línea. Sus características y funcionalidad serán las mismas que las realizadas en contextos presenciales; si bien las diferencias fundamentales se encuentran en las posibilidades que ofrecen los entornos virtuales para favorecer un contexto interactivo tanto con la información como entre los diferentes participantes de la acción formativa, profesorado y alumnado. Al mismo tiempo, la red permite que el alumnado desarrolle tanto actividades individuales como grupales y colaborativas, e interaccionar en un entorno multimedia e hipertextual (Cabero, 2014).

Respecto a sus funciones, debe quedar claro que la importancia de las acciones formativas en red no se encuentra tanto en los contenidos e información que se le presenten a los estudiantes, sino en la interactividad que se pone en funcionamiento para que el proceso de aprendizaje no se convierta en una acción pasiva y memorística. Como indican Cabero y Gisbert (2005, p. 92),

si no queremos convertir los entornos de formación en red en entornos puramente expositivos de bloques de datos y de información, ante los cuales el alumno lo único que debe hacer es memorizar la información que se le presenta, se deben incluir una serie de actividades con las cuales persigamos diferentes objetivos que vayan desde la comprensión de los contenidos, la transferencia a otras situaciones y hechos diferentes a los presentados, o la profundización en los mismos.

De todas formas, muchos docentes siguen considerando que son los contenidos el aspecto al que más significación le conceden para la realización de entorno virtuales formativos de calidad (Boumadan et al., 2020). Por el contrario, como señala Ruiz-Morales (2020, p. 264), "las evidencias sugieren que las prácticas de la instrucción más efectivas incluyen una planificación más concienzuda de las tareas que realizarán los estudiantes y la provisión de feedback mientras las realizan”. Por otra parte, si las e-actividades que diseña el profesor son del tipo colaborativo, ello repercute beneficiosamente en el aumento de la motivación en el estudiante y en la disminución del nivel de frustración (Gómez-Rey et al., 2018).

De igual forma, como señala Silva (2017, p. 7), 
el aprendizaje centrado en las actividades sitúa al estudiante en el centro del proceso formativo, le entrega un rol protagónico, favorece el aprendizaje colaborativo y autónomo. Estas últimas habilidades de orden superior demandadas por la sociedad del conocimiento y útiles no tan solo para la vida académica sino también para la profesional.

En este sentido, para Cabero (2013), las funciones que desempeñan las e-actividades son diversas:

1. Clarificación de los contenidos.

2. Transferencia de la información a contextos y escenarios diferentes en los cuales fueron presentados.

3. Profundización en la materia.

4. Adquisición de vocabulario específico.

5. Socialización.

6. Aplicación de los contenidos a su actividad profesional.

Rodríguez e Ibarra (2011) afirman que, para que las tareas puedan considerarse como auténticas, deben poseer una serie de características que los autores citados concretan en las siguientes:

1. Centrarse en los procesos (no sólo productos).

2. Ser realistas, retadoras, diversas, globales.

3. Ser relevantes, útiles, significativas, complejas.

4. Integrar diferentes competencias, destrezas, habilidades y conocimientos,

5. Implicar diferentes tipos de aprendizaje y actuaciones de forma que faciliten un aprendizaje útil, significativo y estratégico.

6. Facilitar el empoderamiento del estudiante sobre su propio aprendizaje y evaluación.

7. Permitir la construcción del conocimiento y su demostración en contextos reales.

8. Evaluar al estudiante en los contextos profesionales reales.

9. Posibilitar la participación en el proceso de evaluación.

10. Facilitar la proalimentación.

11. Utilizar múltiples medios e instrumentos de evaluación.

12. Proximidad ecológica.

Por otra parte, el diseño de actividades auténticas por parte del docente, y su evaluación continua, favorece el aprendizaje reflexivo y colaborativo (Luo et al., 2017). En consecuencia, no se debe olvidar que las e-actividades que se desarrollan con el alumnado pueden servir para otras funciones además de las indicadas; en concreto, pueden ser de verdadera utilidad para llevar al alumnado para que aprendan a aprender, servir de puente para llevar a cabo un procesamiento profundo 
de la información o profundizar de forma voluntaria en los conceptos que han sido presentados.

La importancia concedida a las e-actividades en el proceso formativo llevan a Sancho y Borges (2011) a diferenciar entre:

1. Aprendizaje centrado en el contenido.

2. Aprendizaje centrado en las actividades.

En la tabla 1 se presentan las características distintivas en cada una de las propuestas.

\section{Tabla 1}

Características del modelo de diseño centrado en contenidos y en actividades.

\section{Centrado en el contenido}

1. El estudiante suele ser reactivo y pasivo, a la espera de lo que diga o decida el docente.

2. El margen de decisión del estudiante es pequeño.

3. Se fomenta un aprendizaje individual.

4. Los estudiantes no tienen muchas oportunidades para aprender autónomamente.

5. Desarrollo de competencias memorísticas y de replicación de contenidos.

6. La educación personal y profesional a menudo está restringida a períodos determinados de la vida.

\section{Centrado en las actividades}

1. Los estudiantes tienen una implicación activa en su aprendizaje, sin esperar que el docente decida por ellos.

2. Mucha libertad para los estudiantes y espacio para las propias decisiones en cuanto a ciertos elementos importantes de su aprendizaje.

3. Se fomenta un aprendizaje en colaboración con los compañeros.

4. Los estudiantes tienen ocasiones de ser autónomos en su aprendizaje.

5. Competencias relacionadas con procesos, con una orientación a resultados, y a la búsqueda, selección y manejo de información.

6. Educación personal y profesional a lo largo de la vida.

Conjuntamente, en su ejecución deben contemplarse las limitaciones que presentan las e-actividades. Según Barberà (2004), son:

1. Dependencia de un ordenador y de la conexión a la red y la falta de acceso por cualquier otro motivo.

2. Ser una copia excesivamente fiel a las actividades presenciales, por lo que no parece necesario su desarrollo.

3. Inferioridad de condiciones por la propia costumbre de desarrollo en el ámbito escolar respecto de las actividades presenciales. 
4. Falta de formación virtual previa tanto por parte de los profesores como por parte de los alumnos.

Para Cabero (2013), el profesor debe tener en cuenta diversos principios generales en su puesta en acción:

1. Variabilidad: deben implicar una diversidad de tipología a e-actividades a realizar por los estudiantes de manera que se potencien con ella la adquisición y evaluación de diferentes tipos de competencias. Tal variabilidad debe entenderse también de la movilización de diferentes tipos de recursos (clip de vídeo, PDF, podcast de audio, objetos en realidad aumentada...) que deben ser movilizados o utilizados por los estudiantes para su realización.

2. De la facilidad a la dificultad: establecer un nivel progresivo de dificultad de las actividades de manera que dé tiempo al alumnado para acostumbrarse a los requerimientos de la acción formativa. Ello permitirá que los estudiantes vayan adquiriendo progresivamente confianza a lo largo del curso, no abandonen por considerar desde el comienzo el curso muy complejo y se familiarice con el proceso evaluativo.

3. La racionalidad temporal: uno de los errores que los alumnos suelen cometer es no valorar con suficiente claridad el tiempo concedido para realizar las acciones formativas.

4. Tanto para lo individual como grupal: proponer actividades que le lleven al estudiante a ejecutar e-actividades, tanto de forma individual como grupalmente. De esta forma, también se potencia la adquisición de competencias sociales y emocionales.

5. Dar normas claras para su realización: su resolución debe venir del esfuerzo cognitivo que el estudiante deba hacer para resolver la tarea, y no para comprender lo que se le pida que debe hacer.

6. Detallar los criterios de evaluación: indicar las normas claras mediante las cuales se van a corregir y evaluar las actividades solicitadas puede ser de gran ayuda para evitar problemas futuros. Puede servir al mismo tiempo para dirigir la calidad de la realización de la actividad por parte del estudiante. Desde esta perspectiva, la utilización de rúbricas puede ser un elemento de ayuda.

Ahora bien, la significación de las e-actividades no se encuentra solo en lo apuntado. Como sugiere un estudio, son también un buen elemento para facilitar la transferencia del aprendizaje puesto que "no solo estamos evaluando qué saben los alumnos, sino también que saben hacer con lo que saben" (Ruiz-Morales, 2020, p. 286). Asociada a esta transferencia, también se encuentra su potencial para favorecer la autonomía del alumnado (Llanes y Novillo, 2018).

Otro de los factores que inciden en la calidad de las e-actividades es la variable tiempo; por una parte, porque una de las competencias que debe poseer el alumnado 
para trabajar en estos entornos virtuales de formación, y que adolece muchas veces de ella, es la competencia de autorregulación del aprendizaje. Por otro lado, al perder el docente en estos entornos los principios de unidad a los que está acostumbrado en la docencia presencial (unidad de tiempo, de acción y de espacio), no se muestra eficaz inicialmente para desempeñarse en este tipo de estructuras docentes y evaluativas. Por este motivo, es importante que, a la hora de especificar el tiempo se realice una planificación tan cuidada que permita el suficiente para que a todo el alumnado, se le dé la oportunidad de realizarla con claridad, reflexionar sobre la misma y preparar sus respuestas (Casal Otero y García Antelo, 2018). Ello, sin lugar a dudas, será muy ventajoso sobre todo para los alumnos y alumnas que presentan diferentes limitaciones: formación, manejo instrumental de las tecnologías, dificultad de comprensión y expresión, problemáticas para relacionarse con los demás en las actividades colaborativas...

Como señala Silva (2011, p. 95),

las actividades son el centro del proceso de enseñanza y aprendizaje, tanto en un entorno presencial como virtual. Sin embargo, en este último, al tratarse de un medio donde la comunicación es esencialmente por medio escrito, y donde los participantes acceden en forma asincrónica, se hace necesario recoger bajo algún formato las instrucciones para el desarrollo de la actividad.

Por tanto, a la hora de presentarlas a los estudiantes, se les debe de incluir diferentes aspectos que le sirvan de ayuda y orientación, tanto para su realización, como para saber la forma en la cual debe cumplimentarla y presentarla, así como también para que conozca los criterios que se manejarán para su evaluación y calificación.

Otra de las variables clave que inciden en el éxito de una e-actividad es asegurarse de que el alumnado entiende lo que se le pide. Independientemente de la ubicación de normas claras para la especificación de la actividad, es aconsejable establecer medidas evaluativas para asegurarnos que los estudiantes lo han entendido. En este caso, puede ser de ayuda solicitar a cualquier estudiante que explique en el foro o un cuestionario lo que se les solicita.

En este sentido, una diversidad de criterios a utilizar en su presentación puede ser de ayuda para facilitar su cumplimentación por los estudiantes (Cabero, 2013). Entre ellos, se pueden señalar los siguientes:

1. Nombre de la e-actividad y unidad a la que pertenece.

2. Objetivos que se persigue que alcancen con su realización.

3. Descripción de la e-actividad: especificar con claridad el contexto y el entorno donde se debe realizar la e-actividad, y dejar perfectamente claro los límites temporales que se piensan asumir para realizarla y entregarla. 
4. Señalar la forma de envío al docente: correo electrónico, tarea en plataforma virtual, publicación en redes sociales...

5. Recursos que debe utilizar para su realización: materiales, documentos, direcciones web...

6. El número de participantes que pueden realizar la actividad y la modalidad de participación (individual, grupal, reparto de trabajo, trabajo colaborativo...) que se permite.

7. Tiempo aproximado que le debe llevar la realización de la e-actividad.

8. Criterios que se utilizarán para valorar la ejecución de la actividad: calificación, ponderación de criterios, e-rúbrica, feedback...

9. Formas en las cuales se deberá de presentar la realización de la actividad: estructura, tipo de material, tamaño/volumen, composición...

10. Fecha de entrega.

Por lo que se refiere a su evaluación, un elemento que puede ser de gran utilidad es la utilización de e-rúbricas que contemplen diferentes dimensiones como:

1. Contenido.

2. Claridad de la respuesta (producto/esquema/actividad/texto) ofrecida.

3. Comprensión de la actividad.

4. Profundización de la respuesta.

5. Expresión clara y correcta.

6. Reflexión.

7. Facilidad de comprensión.

8. Organización de la información y del producto elaborado.

9. Adecuación de la respuesta.

10. Argumenta la reflexión y usabilidad del producto elaborado.

La investigación llevada a cabo por Gros Salvat (2018) pone en énfasis la necesaria evaluación del denominado diseño pedagógico de las e-actividades; el éxito de la misma depende en gran medida de "la capacidad del estudiante para dirigir y gestionar su propio proceso de aprendizaje, estableciendo objetivos y estrategias adecuadas para alcanzar sus objetivos" (p. 74). En este mismo sentido, distintos estudios avalan la necesidad de establecer criterios de evaluación en los que no solo se contemplen indicadores relacionados con la calidad técnica de las e-actividades, sino también su calidad pedagógica (Guri-Rosenblit, 2018; Tumino y Bournissen, 2020). De forma complementaria, Lin et al. (2020) mencionan un tercer elemento clave para el éxito de las propuestas formativas en línea, la incorporación de elementos significativos para el alumnado. Es decir, una e-actividad de calidad es aquella diseñada con criterios técnicos y pedagógicos, y está actualizada y adaptada al contexto en el que se va a llevar a cabo. 
Finalmente, frente a la evaluación centrada en sistemas de preguntas corregidas automáticamente, que se limitan a la evaluación de aprendizajes instrumentales y procedimentales, la evaluación realizada a través de e-actividades, debido a su diversidad, permite evaluar competencias relacionadas con la actividad docente. En este sentido, el estudio y análisis de las mismas es relevante pues el nivel que los docentes suelen poseer en competencia digital es bajo (Dzikite et al., 2017). Por ello, es fundamental establecer líneas de acción formativa específicas y adaptadas al contexto profesional en donde el profesorado desarrolla sus labores. El conseguir reducir esta carencia formativa, permitirá una correcta utilización de la tecnología en escenarios de enseñanza-aprendizaje, donde la selección, creación y modificación de e-actividades es una competencia fundamental (Guillén-Gámez et al., 2020). En este sentido, los Marcos de Referencia de la Competencia Digital Docente como DigCompEdu (Redecker y Punie, 2017; Cabero-Almenara y Palacios-Rodríguez, 2020; Cabero Almenara et al., 2020a, 2020b), abogan por desarrollar planes de formación docente centrados en criterios en los que primen los criterios pedagógicos sobre los tecnológicos como:

1. Identificar, evaluar y seleccionar actividades para la enseñanza y el aprendizaje, entender el copyright aplicable y los requerimientos de accesibilidad.

2. Modificar actividades existentes con licencia abierta y otros recursos en los que está permitido. Crear o co-crear nuevos recursos educativos digitales. Considerar el objetivo de aprendizaje específico, el contexto, el enfoque pedagógico y el grupo de estudiantes, al diseñar los recursos digitales y planificar su uso.

3. Organizar actividades digitales y ponerlas a disposición de los estudiantes y resto de la comunidad educativa. Proteger eficazmente los contenidos digitales sensibles. Respetar las normas de privacidad y derechos de autor. Comprender el uso y la creación de licencias abiertas y recursos educativos abiertos, incluyendo su correcta atribución.

4. Integrar dispositivos y recursos digitales en el proceso de enseñanza, a fin de mejorar la eficacia de las prácticas de enseñanza. Adaptar adecuadamente las bases, administrar y orquestar las intervenciones de enseñanza digital. Experimentar y desarrollar nuevos formatos y métodos pedagógicos de instrucción.

5. Utilizar herramientas y servicios digitales para mejorar la interacción con los estudiantes, de forma individual y colectiva, dentro y fuera de la sesión de aprendizaje. Utilizar las tecnologías digitales para ofrecer orientación y asistencia oportuna y específica. Experimentar y desarrollar nuevas formas y formatos para ofrecer orientación y apoyo.

6. Utilizar las tecnologías digitales para fomentar y mejorar las estrategias de aprendizaje colaborativo. Por ejemplo, como base para el intercambio colaborativo en grupo, como herramienta para realizar una asignación colaborativa, o como medio para presentar resultados. 
7. Utilizar las tecnologías digitales para apoyar los procesos de aprendizaje autodirigidos, es decir, para permitir que los estudiantes planifiquen, supervisen y reflexionen sobre su propio aprendizaje, evidencien el progreso, compartan conocimientos y presenten soluciones creativas.

8. Utilizar herramientas digitales para la evaluación formativa y sumativa. Mejorar la diversidad y la idoneidad de los formatos y enfoques de evaluación.

9. Generar, seleccionar, analizar críticamente e interpretar la evidencia digital de la actividad digital, del rendimiento y del progreso de los estudiantes, con el fin de informar la enseñanza y el aprendizaje.

10. Utilizar herramientas digitales para proporcionar retroalimentación puntual y oportuna a los estudiantes. Adaptar adecuadamente las estrategias de enseñanza y proporcionar apoyo orientado, basado en la evidencia generada por las herramientas digitales utilizadas. Ayudar a los estudiantes y padres y madres a entender la evidencia proporcionada por las herramientas digitales y utilizarlas para la toma de decisiones.

11. Asegurar la accesibilidad a los recursos y a las actividades de aprendizaje, para todos los estudiantes, incluyendo aquellos con necesidades especiales. Considerar y responder a las expectativas digitales de los estudiantes, sus habilidades, usos digitales e ideas erróneas, así como las restricciones contextuales, físicas o cognitivas para el uso de herramientas digitales.

12. Utilizar herramientas digitales para atender las diversas necesidades de aprendizaje de los estudiantes. Por ejemplo, permitiéndoles seguir diferentes vías y metas de aprendizaje, ofreciendo enfoques y herramientas alternativas, y permitiendo a los estudiantes avanzar a diferentes velocidades hacia objetivos de aprendizaje individuales.

13. Utilizar herramientas digitales para fomentar el compromiso activo y creativo de los estudiantes con un tema. Utilizar tecnologías digitales para fomentar las competencias transversales y la expresión creativa de los estudiantes. Abrir el aprendizaje a contextos del mundo real, involucrar a los estudiantes en actividades prácticas, en la investigación científica, la resolución de problemas complejos, la expresión creativa.

\section{LAS E-ACTIVIDADES: SU TIPOLOGÍA}

Por lo que se refiere a su tipología, Moreno y Bailly-Baillière (2002) las clasifica en explicaciones en red, presentaciones del alumnado, actividades de repetición, rastreo de información, investigación guiada, análisis guiado, trabajo en equipo, tormenta de ideas, estudios de casos, juegos de rol, crítica de grupo, simulaciones, instrucciones de uso y juegos didácticos.

Stansfieled (1999) agrupa las actividades en dos grandes categorías: personales y sociales. En las primeras incluye: actitud y capacidad para intentar nuevos caminos de hacer las cosas, tomar responsabilidad sobre su propio aprendizaje, interesarse por 
los propios procesos de aprendizaje, reconocimiento de los beneficios y limitaciones de los conceptos tradicionales de conocimiento y comprensión y sus conexiones con su experiencia, estilo de aprendizaje activo, ansias para el conocimiento, la comprensión y la experiencia, favorecer un proactivo y entusiástico aprendizaje. En las segundas: habilidad para ver la relevancia de su desarrollo en un contexto más amplio de ellos (percepciones de los valores de los otros), habilidad para captar en su amplio contexto el poder pensar fuera del contexto escolar, desear el desarrollo de los demás, buena disposición para la participación, la habilidad para admitir el valor intrínseco de las contribuciones de sus pares, preparación para poner un esfuerzo extra y para seguir las instrucciones de los tutores, y la habilidad para ejercer el pensamiento independiente y restringir la confianza. En una línea muy similar en nuestro contexto, Barberà (2004) las clasifica en función de que puedan ejecutarse de forma individual (autoaprendizaje electrónico, cubículos virtuales, aprendizaje cognitivo, autorización inteligente, laboratorios virtuales, y proyectos telemáticos) o grupal (proyectos telemáticos, grupos cooperativos, círculos de aprendizaje, debates, y comunidades virtuales de aprendizaje).

Por su parte, Alonso y Blázquez (2012) discriminan entre e-actividades para la búsqueda de información (caza del tesoro y webquest) y para la elaboración de la información (base de datos, resumen, esquema, mapas conceptuales, elaboración de multimedia, podcast, elaboración de imágenes y creación de vídeos).

Marcelo et al. (2019) diferencia entre actividades asimilativas (persigue la comprensión de la información por parte del estudiante), productivas (elaboración de un producto por el estudiante), de gestión de la información (actividades de búsqueda, síntesis y presentación de un producto sobre lo realizado), comunicativa (discusión sobre la información), de aplicación (resolución de una situación problemática), experiencial (ubicación del estudiante en un contexto real laboral) y evaluativa (analizar las competencias adquiridas por los estudiantes).

Maina (2020) señala que hay diferentes tipos de e-actividades que se pueden poner en funcionamiento en la formación en red, que concreta en las siguientes:

1. Actividades de análisis y síntesis.

2. Actividades de investigación o resolución de problemas.

3. Actividades de interacción y comunicación.

4. Actividades de construcción colaborativa de conocimiento.

5. Actividades de reflexión.

Abordando ya la diversidad de tipología que podemos utilizar como instrumentos de evaluación, desde el principio son inicialmente las mismas que se pueden derivar de la enseñanza presencial, ampliándolas con aquellas específicas que la red aporta, y que las herramientas de la web 2.0, nos permiten poner en funcionamiento. Al respecto, se encuentran diferentes propuestas realizadas por distintos autores e instituciones. Así, la Universidad de Maryland (2004) ha elaborado un sitio web 
para ayudar a los profesores a diseñar los sitios y ubicar diferentes actividades. En estas últimas, señala como las pertinentes a realizar las siguientes: aprendizaje conceptual, resolución de problemas, análisis de objetos y documentos, recoger datos y sintetizarlos, estudio de casos, presentaciones por profesores y por estudiantes, aprendizaje colaborativo, realización de investigaciones y laboratorio virtual y visitas de campo.

Por su parte, Moreno y Bailly-Baillière (2002) señalan las siguientes actividades que se pueden utilizar en la formación virtual: explicaciones en red, presentaciones de los alumnos, actividades de repetición, rastreo de información, investigación guiada, análisis guiado, trabajo en equipo, tormenta de ideas, estudios de casos, juegos de rol, crítica de grupo, simulaciones, instrucciones de uso y juegos didácticos.

Harris (1995), en su "problem-solving projects", establece diferentes tipos de actividades que pueden ser realizadas por los estudiantes: búsqueda de información (a los estudiantes se les presentan diferentes problemas y deben identificar información para resolverlos), procesos escritos electrónicos (los estudiantes realizan ensayos y poemas y reciben las sugerencias de otra serie de compañeros), creación de secuencias (dibujan un documento), resolución paralela de problemas (diversos estudiantes trabajan de forma independiente para resolver un problema), reuniones virtuales (chat), simulaciones (trabajo colaborativo en línea para recrear un evento, diseño de contextos, manipulación de variables,...), proyectos de acción social...

Barberà (2004), desdela clasificación que realiza de las actividades en individuales y grupales, propone la siguiente lista para las realizadas de forma individual: autoaprendizaje electrónico, cubículos virtuales, aprendizaje cognitivo, autorización inteligente, laboratorios virtuales y proyectos telemáticos. Además, las siguientes para las ejecutadas de forma grupal: proyectos telemáticos, grupos cooperativos, círculos de aprendizaje, debates y comunidades virtuales de aprendizaje.

En el documento elaborado por la Dirección de Educación Superior (2020) del Ministerio de Cultura y Educación del Gobierno de Formosa de Argentina denominado "Los procesos de evaluación en entornos virtuales de aprendizaje" también se proponen una diversidad de ellas: diarios de reflexión, dianas, portafolio, mapa conceptual como organizador gráfico, organizadores gráficos, cuestionarios interactivos, producciones grupales en línea, foros de debate o listas de discusión, diario, vídeos, formularios de Google, Edupuzle, Educa Play, Kahoot, y producciones escritas en diferentes formatos.

De la misma forma, diferentes autores han ampliado las diferentes propuestas identificadas anteriormente proponiendo una lista bastante exhaustiva de e-actividades que pueden realizar para la evaluación de los estudiantes en contextos de formación (Cabero, 2014; Colomo Magaña et al., 2020; Barroso, 2017). Se proponen las siguientes: proyectos de trabajo, visitas a sitios webs, realización y construcción de ejemplos, análisis de imágenes y clip de vídeos, estudios de casos, búsqueda adicional de información o de ejemplos, trabajar con un caso para 
resolver un problema y contribuir con materiales adicionales materiales para que el caso sea usado por otros, participar en una situación de juego de roles y guardar los resultados de otros para considerarlos, crear un informe para ser usado como recurso de aprendizaje por otros, crear productos, tales como multimedia o su diseño, que también pueden ser utilizados por otros, extender y aplicar principios teóricos a nuevos escenarios y añadir estos resultados, participar en una discusión y grabar los aspectos claves para ser usados por otros, solución de problemas, análisis de objetos y documentos, reunión de datos y síntesis, estudio de casos, laboratorios virtuales y viajes al campo, solucionario de puzles, preguntas incorporadas en vídeo con programas como Edupuzle o $\mathrm{HP}_{5}$, o aplicaciones de gamificación. De todas formas, no se pretende indicar que con esta lista se acoten las e-actividades que puede utilizar el docente para la evaluación de los estudiantes

\section{LAS E-ACTIVIDADES: SU SELECCIÓN}

Es necesario señalar que los criterios que se pueden movilizar para la selección y determinación de las e-actividades más adecuadas a nuestra situación son diversos, y algunos de ellos pueden ser los siguientes:

1. Pensar en las actividades más usuales que se pueden desarrollar en el campo científico-temático en el cual nos desenvolvemos.

2. Tener presente las características de los alumnos: dominio del vocabulario, edad, experiencia profesional, situación laboral...

3. Plantear actividades que puedan movilizar en el estudiante diferentes tipos de capacidades, y permitan alcanzar una diversidad de competencias.

4. Contemplar en la selección las actividades que sean más motivantes para los alumnos.

5. Tener en cuenta las posibilidades que la red ofrece para su desarrollo y cumplimentación.

Al mismo tiempo, puede ser de ayuda para su determinación apoyarse bien en la propuesta que Churches (2009) realizó para adaptar la taxonomía de Bloom a la era digital, donde ya la última categoría no es "evaluar" si no la de "crear". De la misma forma, la rueda de la pedagogía de Allan Carrington (2014), construida para que los estudiantes realicen actividades para analizar, aplicar, recordar-comprender, crear y evaluar. Estas propuestas son de ayuda para solucionar uno de los problemas apuntados por Barberà $(2004$, p. 45), cuando llama la atención respecto que la "mayor parte de las e-actividades que encontramos son de naturaleza simple y no aportan al formato de lápiz y papel más que la sistematización y la posibilidad de repetición hasta la saciedad y de transmisión electrónica al propio profesor”. De esta forma, se consigue favorecer un aprendizaje activo, autónomo, constructivo y 
colaborativo, y nuevas modalidades de evaluación no centradas en la memorización y recuerdo de la información por parte del estudiante.

Finalmente, se señala la fuerte relación que se presenta entre la evaluación mediante e-actividades y la construcción de portafolios electrónicos, que recopilen la diversidad de trabajos que ha ido realizando un estudiante a lo largo de un período de tiempo formativo concreto (Blázquez et al., 2017).

\section{REFERENCIAS}

Alonso, L., y Blázquez, F. (2012). El docente de educación virtual. Guía básica. Narcea.

Barberà, E. (2004). La educación en red. Paidós.

Barrientos-Hernán, E., López-Pastor, V., y Pérez-Brunicardi, O. (2020). Evaluación Auténtica y Evaluación Orientada al Aprendizaje en Educación Superior. Una Revisión en Bases de Datos Internacionales. Revista Iberoamericana de Evaluación Educativa, 13(2), 67-83. https://doi.org/10.15366/ riee2020.13.2.004

Barroso, J. (2017). Diseño, producción, evaluación y utilización educativa de la Realidad Aumentada. Secretariado de Recursos Audiovisuales y Nuevas Tecnologías Universidad de Sevilla.

Blázquez, F., Alonso, L., y Yuste, R. (2017). La evaluación en la era digital. Síntesis.

Boumadan, M., Soto-Varela, R., OrtizPadilla, M., y Poyatos-Dorado, C. (2020). What Factors Determine the Value of an Online Teacher Education Experience from a Teacher's Perspective? Sustainability, 12, 8064. https://doi. org/10.3390/su12198064

Brown, S. (2015). La evaluación auténtica: el uso de la evaluación para ayudar a los estudiantes a aprender. Relieve, 1(2). https://doi.org/10.7203/ relieve.21.2.7674

Cabero, J. (2013). La formación virtual en el nuevo entramado de la web 2.o: el e-learning 2.o. Alianza.
Cabero, J. (2014). Manual para el Desarrollo de la formación virtual en el Instituto Tecnológico de Santo Domingo. Intec.

Cabero, J., y Gisbert, M. (2005). Laformación en Internet. Guía para el diseño de materiales didácticos. Eduforma.

Cabero-Almenara, J., Barroso-Osuna, J., Palacios-Rodríguez, A., y Llorente-Cejudo, C. (2020a). Marcos de Competencias Digitales para docentes universitarios: su evaluación a través del coeficiente competencia experta. Revista Electrónica Interuniversitaria De Formación Del Profesorado, 23(2). https://doi. org/10.6018/reifop.413601

Cabero-Almenara, J., Romero-Tena, R., y Palacios-Rodríguez, A. (2020b). Evaluation of Teacher Digital Competence Frameworks Through Expert Judgement: the Use of the Expert Competence Coefficient. Journal of New Approaches in Educational Research, 9(2), 275293. $\quad$ http://dx.doi.org/10.7821/ naer.2020.7.578

Cabero-Almenara, J., y Palacios-Rodríguez, A. (2020). Marco Europeo de Competencia Digital Docente «DigCompEdu». Traducción y adaptación del cuestionario «DigCompEdu Check-In». EDMETIC, 9(1), 213-234. https://doi.org/10.21071/ edmetic.vgi1.12462

Cabrera, N., y Fernández-Ferrer, M. (2020), Claves para una evaluación en línea. En Sangrá, A. (coord.), Decálogo para la mejora de la docencia on-line (81-98). UOC. 
Carrington, A. (2014). La rueda de la pedagogía (Pedagogy Wheel) de Allan Carrington. Learn.

Casal Otero, L., y García Antelo, B. (2018). Una experiencia de formación del profesorado para implementar la evaluación entre pares en el campus virtual de la Universidad de Santiago de Compostela. Píxel-Bit. Revista de Medios y Educación, 54, 125-144. https://doi. org/10.12795/pixelbit.2019.i54.07

Churches, A. (2009). Taxonomía de Bloom para la era digital. Eduteka.

Colomo Magaña, E., Gabarda Méndez, V., Cívico Ariza, A., y Cuevas Monzonís, N. (2020). Percepción de estudiantes sobre el uso del videoblog como recurso digital en educación superior. Píxel-Bit. Revista De Medios Y Educación, 59, 7-25. https:// doi.org/10.12795/pixelbit.74358

Cubero-Ibáñez, J., y Ponce-González, N. (2020). Aprendiendo a través de Tareas de Evaluación Auténticas: Percepción de Estudiantes de Grado en Educación Infantil. Revista Iberoamericana de Evaluación Educativa, 13(1), 41-69. https://doi.org/10.15366/ riee2020.13.1.002

Díaz Rosas, F., Cuevas López, M., Fernández Cruz, M., Gijón Puerta, J., Lizarte Simón, E. J., Ibáñez Cubillas, P., El Homrani, M., Ávalos Ruiz, I., y Rodríguez Muñoz, R. J. (2019). Liderazgo y calidad en la Educación Superior. EDMETIC, 8(2), 52-72. https://doi.org/10.21071/edmetic. v8i2.12120

Dirección de Educación Superior (2020). Los procesos de evaluación en entornos virtuales de aprendizaje. Ministerio de Educación y Cultura del Gobierno de Formosa. Gobierno de Argentina.

Dzikite, C., Nsubuga, Y., y Nkonki, V. (2017). Lecturers' Competencies in Information and Communication Technology (ICT) for Effective Implementation of ICTIntegrated Teaching and Learning in Textiles and Clothing Degree Programmes.
International Journal of Educational Sciences, 17(1-3), 61-68. https://doi.org/ 10.1080/09751122.2017.1305756

Fuentes-Guerra, M., y García, M. (2003). Los procesos de planificación de la Universidad. Unidad para la Calidad de las Universidades Andaluzas.

Gómez-Rey, P., Barberà, E., y FernándezNavarro, F. (2018). Students' perceptions about online teaching effectiveness: A bottom-up approach for identifying online instructors' roles. Australasian Journal of Educational Technology, 34(1), 116130. https://doi.org/10.14742/ajet.3437

Gros Salvat, B. (2018). La evolución del e-learning: del aula virtual a la red. RIED. Revista Iberoamericana de Educación a Distancia, 21(2), 69-82. https://doi. org/10.5944/ried.21.2.20577

Guillén-Gámez, D., Ruiz-Palmero, J., Sánchez-Rivas, E., y Colomo-Magaña, E. (2020). ICT resources for research: an ANOVA analisis on the digital research skills of higher education teachers comparing the areas of knowledge within each gender. Education and Information Technologies, 25, 4575-4589. https://doi. org/10.1007/s10639-020-10176-6

Guri-Rosenblit, S. (2018). E-Teaching in Higher Education: An Essential Prerequisite for E-Learning. Journal of New Approaches in Educational Research, 7(2), 93-97. http://doi. org/10.7821/naer.2018.7.298

Harris, J. (1995). Educational telecomputing activities: problem-solving projects. Learning and leading with technology, 22(8), 59-63.

Lai, P., Portelese, A., y Jacobson, M. (2017). Does sequence matter? Productive failure and designing online authentic learning for process engineering. British Journal of Educational Technology, 48, 6, 12171227. https://doi.org/10.1111/bjet.12492

Lezcano, L., y Vilanova, G. (2017). Instrumentos de evaluación de aprendizaje en entornos virtuales. 
Perspectiva de estudiantes $y$ aportes de docentes. ICT-UNPA. https://doi. org/10.22305/ict-unpa.v9i1.235

Lin, Q., Yin, Y., Tang, X., Hadad, R., y Zhai, X. (2020). Assessing learning in technologyrich maker activities: A systematic review of empirical research. Computers \& Education, 157, 103944. https://doi. org/10.1016/j.compedu.2020.103944

Llanes, J., y Novillo, P. (2018). Virtual Master's Training: Implications for Teaching. Journal of Academic Perspectives, 3, 1-36.

Luo, T., Murray, A., y Crompton, H. (2017). Designing Authentic Learning Activities to Train Pre-Service Teachers About Teaching Online. International Review of Research in Open and Distributed Learning, 18(7), 141-156. https://doi. org/10.19173/irrodl.v18i7.3037

Maina, M. (2020). E-actividades para un aprendizaje activo. En A. Sangrá (coord.), Decálogo para la mejora de la docencia on-line (81-98). UOC.

Marcano, B., Íñigo, V., y Sánchez Ramírez, J. M. (2020). Validación de rúbrica para evaluación de e-actividades diseñadas para el logro de competencias digitales docentes. Apuntes Universitarios, 10(2), 115-129. $\quad$ https://doi.org/10.17162/ au.v10i2.451

Marcelo, C., Murillo, P., y Marcelo, P. (2019). Diseño de módulos en líneas enriquecidos con tecnologías digitales. UTP.

Medina-Díaz, M., y Verdejo-Carrión, A. (2020). Validez y confiabilidad en la evaluación del aprendizaje mediante las metodologías activas. Alteridad, 15(2), 270-283. https://doi.org/10.17163/alt. v15n2.2020.10.

Moorhouse, B. L. (2020). Adaptations to a face-to-face initial teacher education course 'forced' online due to the COVID-19 pandemic. Journal of Education for Teaching. https://doi.org/10.1080/0260 7476.2020.1755205
Moreno, F., y Bailly-Bailleère, M. (2002). Diseño instructivo de la formación online. Aproximación metodológica a la elaboración de contenidos. Ariel.

Padilla-Hernández, A. L., Gámiz-Sánchez, V. M., y Romero-López, M. A. (2019). Niveles de desarrollo de la Competencia Digital Docente: una mirada a marcos recientes del ámbito internacional. Innoeduca. International Journal of Technology and Educational Innovation, 5(2), 140-150. https://doi.org/10.24310/ innoeduca.2019.v5i2.5600

Redecker, C., y Punie, Y. (2017). Digital Competence of Educators DigCompEdu. Publications Office of the European Union.

Rodríguez. G., e Ibarra, M. S. (2011). E-Evaluación orientada al e-aprendizaje estratégico en educación superior. Narcea.

Ruiz-Morales, Y. A. (2008). La evaluación educativa y su impacto en el rendimiento académico de los estudiantes universitarios. Encuentro educacional, 15(2), 230-239.

Ruiz-Morales, Y. A. (2020). Evaluación formativa y compartida para el desarrollo de competencias investigativas en estudiantes universitarios. Educere.

Sancho, T., y Borges, F. (2011). El aprendizaje en un entorno virtual y su protagonista, el estudiante virtual. UOC.

Sangrá, A. (2020). Decálogo para la mejora de la docencia on-line. UOC.

Silva, J. (2011). Diseño y moderación de entornos virtuales de aprendizaje (EVA). UOC.

Silva, J. (2017). Un modelo pedagógico virtual centrado en las E-actividades. RED. Revista de Educación a Distancia, 53. https://doi.org/10.6018/red/53/10

Stansfieled, L. M. (1999). Capabilities for successful self-development. Kogan.

Tumino, M. C., y Bournissen, J. M. (2020). Integration of ICT in the classroom and its impact on students: construction and 
validation of measurement scales. IJERI: International Journal of Educational Research and Innovation, (13), 62-73. https://doi.org/10.46661/ijeri.4586
Universidad de Maryland (2004). Using the Web to Design Online Courses. UMUC.

\section{PERFIL ACADÉMICO Y PROFESIONAL DE LOS AUTORES}

Julio Cabero-Almenara. Adscrito al Departamento de Didáctica y Organización Educativa de la a la Facultad de Ciencias de la Educación. Es director del Grupo de Investigación Didáctica (GID-HUM 390), director del Secretariado de Recursos Audiovisuales y Nuevas Tecnologías, editor jefe de la revista Pixel-Bit. Revista de Medios y Educación y miembro fundador de Edutec. Ha participado en numerosas publicaciones y cursos relacionados con la Tecnología Educativa. https:// orcid.org/0000-0002-1133-6031

E-mail: cabero@us.es

Antonio Palacios-Rodríguez. Graduado en Educación Primaria por la Universidad de Sevilla. También ha realizado el Máster Universitario en Dirección, Evaluación y Calidad de las Instituciones de Formación. Es miembro del Grupo de Investigación Didáctica (GID-HUM 390): Análisis Tecnológico y cualitativo. Actualmente trabaja como Contratado Predoctoral FPU en el Departamento de Didáctica y Organización Educativa de la Facultad de Ciencias de la Educación, Universidad de Sevilla. Su experiencia docente e investigadora está relacionada con la Tecnología Educativa y la formación del profesorado. https://orcid.org/oooo0002-0689-6317

E-mail: aprodriguez@us.es

\section{DIRECCIÓN DE LOS AUTORES}

Facultad de Ciencias de la Educación de la Universidad de Sevilla

Calle Pirotecnia s/n

41018-Sevilla (España

Fecha de recepción del artículo: 29/11/2020

Fecha de aceptación del artículo: 25/01/2021

Fecha de aprobación para maquetación: 11/02/2021 